\title{
EDITORIAL
}

\section{Good things come in small packages: exosomes, immunity and}

\section{cancer}

Cancer Gene Therapy (2014) 21, 139-141; doi:10.1038/cgt.2014.14

"Think of the toddler years as a wacky and crazy - but short period of time,... see past the chaos of it, and you'll see that good things truly do come in small packages." - Paula Spencer Scott

Cancer, the second leading cause of death worldwide and by 2020 thought to be the first in the Western world, is a complex group of diseases categorized by unscheduled cell death, reparative cellular proliferation, a host wound healing response, and development of chaotic genomic instability arises from a single cell, at least based on our current understanding-indeed a small package. Unlike traditional surgical, medical and irradiation therapies, modern gene therapy provides a new platform for targeting cancer based on its molecular characteristics. Gene therapy can directly target tumor cells by delivering therapeutic DNAs, microRNAs (miRNA) and small interfering RNAs into cancer cells to alter cellular physiology or alternatively to promote an effective targeted immune response. Although this can be accomplished most efficiently using recombinant viruses including lentiviruses, adenoviruses, vaccinia and herpes simplex virus with specific DNA or miRNAs, direct injection of naked DNA, physical delivery methods including gene gun, electroporation and laser and chemical treatments with lipids and inorganic particles can also deliver small snippets of DNA.

Indeed, two decades following our initiation of gene therapy trials while at the National Institutes of Health, more than 1800 clinical trials have been applied worldwide. Some of these approaches actually provide therapeutic benefits to patients with glioblastoma, ovarian cancer, lymphoma and hepatocellular carcinoma. However, there are still substantive challenges for the extensive application of gene therapy in the clinic. The twin Achilles heels include the efficiency and specificity of gene transfer and the second, the immune response, particularly to viral vectors. ${ }^{1}$ What's more, large viruses with their intrinsic ability to trigger innate immune mechanisms may cause damage to normal tissues and organs, representing a major safety concern. Arising as potential natural vehicles for gene delivery are so called 'exosomes'.

Exosomes are $30-100 \mathrm{~nm}$ membrane vesicles derived from the late endocytic compartment and released into the extracellular space by fusion of multivesicular endosomes with the cell membrane. Although they were first found in maturing red blood cells, these vesicles can be generated by a large number of cell types including tumor cells, neurons and immune cells and so on, and also detected in body fluids such as serum and plasma. Typical exosomes express major histocompatibility complex (MHC) I and MHC II on their surface and contain specific marker tetraspanins (CD9, CD63, CD81), heat-shock proteins, lipids and miRNAs. miRNAs are small (19-22 nt), non-coding RNAs capable of regulating a variety of target genes at the posttranscriptional level. Many miRNAs are aberrantly expressed in malignant cells and the individual miRNA content between exosomes derived from cancer patients and normal individuals now has the potential to distinguish these two states, contributing to better diagnosis, enabling tumor progression determination and further treatment. The fully explicated biological function of exosomes in vivo remains unclear; however, these nanovesicles seem to be involved in cell-cell communication, serving as 'tweets' between cells to drive an emergent anti-tumor immune response, and could even be considered as components of cancer vaccines. Recent studies suggest that miRNA in exosomes can be transferred between cells to initiate anti-tumor immune response and inhibit tumor cell proliferation.

\section{Exosomes as delivery vehicles for miRNA}

Some specific miRNAs are downregulated in tumor-bearing patients and restoration of the expression of these tumor suppressor miRNAs can inhibit tumor progression, but the efficiency of traditional gene therapy strategies is not high enough for clinical application. miRNAs found within exosomes present the possibility that miRNAs are involved in cell-cell communication as humoral factors and that exosomes themselves could function as 'natural' delivery vehicles for gene therapy. This possibility is confirmed by the fact that neighboring dendritic cells (DCs) can contact each other and regulate the cell maturation state and physiological function using such exosome-shuttle miRNAs. Exosomes generated by DCs can fuse with the target DC membrane and release the contents into the cytosol including the functional miRNA, which can posttranscriptionally repress target mRNAs of the acceptor DC. ${ }^{2}$ Astonishingly, exosomes from marrow stromal cells transfected with miR-146b expression plasmids can significantly suppress glioma xenograft growth of primary brain tumors in a rat model following intra-tumoral injection, ${ }^{3}$ which suggests that exosomes indeed have the ability to transfer miRNA to tumor cells as vectors for gene therapy. Apart from functioning as delivery vesicles, exosomes isolated from normal cell lines can also inhibit the proliferation of tumor cells. ${ }^{4}$ Exosomes derived from a cancerous prostate cell line contain lower levels of miR-16, -143 and -205 than those from a noncancerous prostate epithelial cell line. Application of exosomes from normal cells could restore the tumor suppressor miRNAs within cancer cells and inhibit cellular proliferation. This anti-tumor effect can be abolished by neutralizing miRNA with an anti-miR-143 antibody, suggesting that miR-143 has an important role in cancer suppression and accidental loss of specific miRNAs may indeed be associated with tumor initiation. Most importantly, exogenously transduced miR-143 in exosomes has no effect on the proliferation of non-cancerous cells. Thus, excessive numbers of tumor suppressor miRNAs do not inhibit normal cell growth, even in the presence of physiological levels of other miRNAs. In addition, no obvious side effects have been found in exosome-mediated gene delivery in vivo. But what about immunity?

miRNAs targeting Toll-like receptors miRNAs can be released in exosomes for transfer to acceptor cells and to regulate cellular protein expression by seeding sites located within the $3^{\prime}$ UTR of mRNA. This imperfect complementary binding results in the translational repression of target mRNAs and 
speeds up mRNA degradation via deadenylation and resultant gene silencing. Surprisingly, it has been recently demonstrated that miRNAs can directly interact with proteins including Toll-like receptors (TLRs) to drive an immune response. TLRs are a family of receptors that enable innate immune cells and other specialized cell types including epithelial cells to recognize pathogenassociated molecular pattern molecules and protect the host from pathogens. TLRs have 10 members in human (13 in murine), some of which are located intracellularly within the endosome (human TLR8 and murine TLR7). These TLRs can bind single-strand RNA (ssRNA) sequences in the cytosol and lead to cell activation and cytokine production dependent on signaling through the

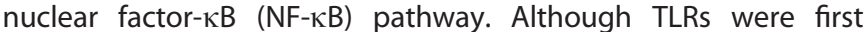
identified on immune cells, malignant epithelial cells can also express TLRs, which have discordant effects on tumor development. Within the tumor microenvironment, the activation of TLRs can inhibit the tumor growth by induction of tumor cell apoptosis or stimulation of anti-tumor immune responses. Alternatively, this activation can favor tumor generation, development and escape from immunity by promoting chronic inflammation. For example, non-small-cell lung cancer (NSCLC) cell lines release high levels of miR-21 and miR-29a intracellularly and extracellularly when compared with normal cell lines. Both of them can functionally interact with TLRs on macrophages and induce increased production of the proinflammatory and prometastatic cytokines interleukin (IL)- 6 and tumor necrosis factor- $\alpha$ (TNF $\alpha$ ), which increases the metastatic potential of Lewis lung carcinoma cells. These effects are dependent on exosomal miRNA interaction with murine TLR7. When TLR7 is knocked out or when mice are treated with exosome-lysosome fusion or exosome release inhibitors, the secretion of TNF $\alpha$ and IL- 6 are reduced and there are fewer tumors. $^{5}$ Except for stimulating TLR7 to promote tumor progression, miRNAs can also induce innate immune response via nature killer (NK) cell activation in a TLR1-dependent signaling pathway. Application of miR-122 and miR-15b, which are highly expressed in exosomes isolated from healthy donor serum, can enhance activation marker CD69 expression, interferon- $\gamma$ production and degranulation marker CD107a expression on human NK cells. What's more, administration of miRNAs in vivo significantly results in NK but not T-cell activation and protects mice from tumor progression in an NK cell-dependent manner. Knockdown of TLR1 blunts miRNA-induced downstream of NF- $\kappa B$ pathway activation, which suggests that miRNAs can inhibit tumor development by selectively activating NK cells via interaction with TLR1. ${ }^{6}$ Thus, miRNAs can mediate tumor growth in a positive or negative manner and the actual effect depends on the individual miRNAs, the TLRs involved and the activated cell types.

\section{Tumor miRNA regulating host biology}

Various stressors including ultraviolet light, chemical agents, chronic acid exposure and viruses can cause damage to normal cells, cell death, replicative restoration and accumulation of genetic mutation, which can promote carcinogenesis. Some cancer cells acquire the capacity to enter the blood and lymphatic vessels and travel to other sites. Each tumor type has preferential organs and thus before metastasis, a premetastatic niche forms in the selected organ and prepares the tissues for the metastatic cells

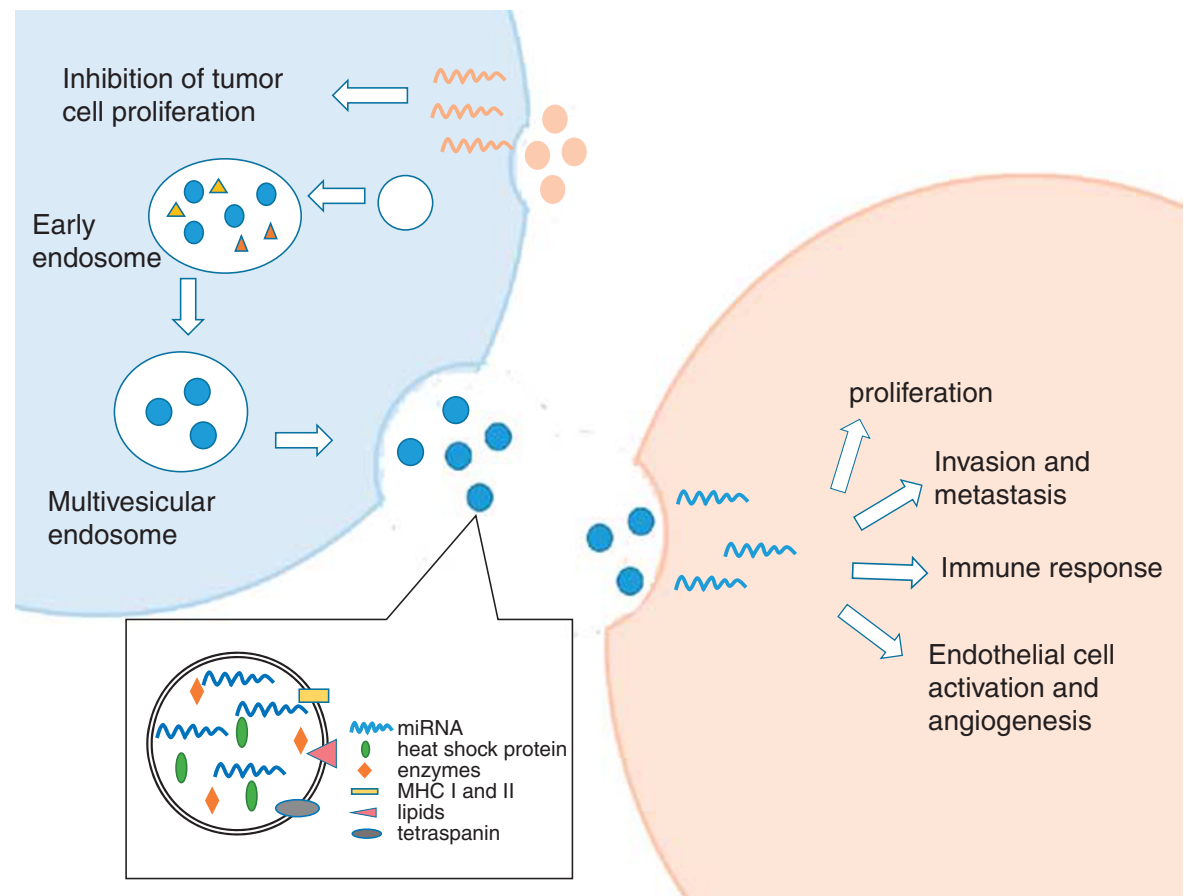

Figure 1. Exosomes are tiny membrane vesicles generated from the late endosome system. Stimulated by extracellular factors, vesicles directly bud from the cell membrane and form early endosomes, which can further develop into multivesicular bodies. These late endosomes can either fuse with the lysosome to degrade external pathogens or release their contents, exosomes, to communicate with other cells. Canonical exosomes express several transmembrane proteins, such as MHC I, MHC II and specific tetraspanins (CD9, CD63 and CD81), and also contain important miRNA molecules and heat-shock proteins. Exsomes derived from tumor cells can regulate a variety of cell functions by fusing with their cell membrane and releasing miRNAs into the cytosol. These exosomal tumor miRNAs can (i) promote neighboring cell proliferation by upregulating cell-cycle proteins, (ii) initiate the formation of a premetastatic niche in target organs to prepare for tumor cell implantation and growth, (iii) induce immune responses via interaction with toll-like receptors, which can have various effects on tumor progression and (iv) activate endothelial cells to speed up tube formation and enhance angiogenesis. Exosomes derived from normal somatic cells can deliver tumor suppressor miRNAs to malignant cells, restoring the deficient expression of these miRNAs in tumor cells and silencing cell cycle- and growth-related mRNAs to suppress tumor progression. Exosomes generated from cells overexpressing suppressor miRNAs via plasmids demonstrate a similar inhibitory effect on tumor development. 
ability to settle and grow. Exosomal tumor miRNA can modulate premetastatic tissues to prepare a premetastatic niche in advance of the tumor cells arrival by significantly influencing the mRNA translation in the target cells. Subcutaneous injection of metastatic rat adenocarcinoma-derived exosomes increases protease activity, prominent adhesion molecules and chemokine ligand expression, upregulation of proliferation-, oxidative stress- and angiogenesisrelated gene expression to fit the demands for metastatic cell hosting and growth. This effect relies on the expression of high amounts of both miR-494 and miR-542-3p in the metastatic carcinoma-derived exosomes. ${ }^{7}$ In addition to preparing a suitable microenvironment for metastasis, exosomal miRNAs also affects the angiogenic activity of endothelial cells in hypoxic settings. Owing to the rapid growth of tumor cells, the vasculature surrounding and within the tumor cannot provide sufficient oxygen and nutrition for basal metabolic requirements. Indeed, most cancer cells are hypoxic. Exosomes isolated from leukemia cells under hypoxic condition significantly enhance angiogenic tube formation by human umbilical vein endothelial cells (HUVECs) compared with exosomes generated under normoxic conditions. miR-210 in exosomes contributes to the increased angiogenesis and blockade of the exosomal miR-210 with antibody reduces tube formation but not the endogenous HUVECs miR-210. ${ }^{8}$ Tumor cells generate exosomes to deliver their miRNAs to communicate with neighboring endothelial cells and distant organ cells for angiogenesis and metastasis, which provides not only a suitable microenvironment for tumor growth and progression, but also effective biomarkers for diagnosis and specific targets for cancer immunotherapy treatments (Figure 1).

\section{Perspectives}

Cancer in adults usually arises as a consequence of chronic stress and a chronic inflammatory response. ${ }^{9}$ Exosomes have potential as gene therapy vehicles for established cancers or alternatively as cell-free vaccines for cancer immunotherapy. They show remarkable inhibitory effects on tumor cell proliferation and tumor progression in animal models and in early clinical trials. Typical exosomes are able to induce anti-tumor immune responses via several cell surface ligands, with which they interact. They can activate T cells via antigen presentation, NK cells via interaction with NKG2D ligand and DCs via antigen transfer. Moreover, exosomes can function as delivery vehicles for gene therapy and transfer tumor suppressor miRNAs to tumor cells to silence individual mRNAs and regulate the cell cycle both in vivo and in vitro. There are many challenges remaining in the field of exosome research, which involve development of straightforward and reproducible protocols for exosome extraction and the further understanding of exosome biogenesis and function. Fundamental knowledge about how informationcarrying molecules including miRNAs and antigens are packaged and transferred, how miRNAs modulate specific gene transcription and how exosomes and suppressor miRNAs influence normal cell physiological function is still lacking. Large amounts of highly purified, well-characterized exosomes are essential for both animal studies and clinical trials. The efficiency of exosome generation needs to be largely improved. Despite all these obstacles, exosome gene therapy provides another potential cancer treatment by combining gene therapy and immunotherapy. As exosomes for therapy can be produced by patients and exist within body fluids, this treatment is simple, feasible and should not lead to immediate immune response and rejection. Considering the broad application prospects, increased efforts should be made in the coming years to fully understand the biology of exosomes and consider their broader application for tumor gene therapy.

W Wang ${ }^{1,2,3,4}$ and MT Lotze $\mathrm{L}^{1,2,3}$

${ }^{1}$ Department of Surgery, University of Pittsburgh School of Medicine, University of Pittsburgh Cancer Institute, Pittsburgh, PA, USA;

${ }^{2}$ Department of Immunology, University of Pittsburgh School of Medicine, University of Pittsburgh Cancer Institute, Pittsburgh, PA, USA;

${ }^{3}$ Department of Bioengineering, University of Pittsburgh School of Medicine, University of Pittsburgh Cancer Institute, Pittsburgh, PA, USA and

${ }^{4}$ Tsinghua University School of Medicine, Beijing, China E-mail: lotzmt@upmc.edu

\section{REFERENCES}

1 McCormick F. Cancer gene therapy: fringe or cutting edge? Nat Rev Cancer 2001; 1: 130-141.

2 Montecalvo A, Larregina AT, Shufesky WJ, Stolz DB, Sullivan ML, Karlsson JM et al. Mechanism of transfer of functional microRNAs between mouse dendritic cells via exosomes. Blood 2012; 119: 756-766.

3 Katakowski M, Buller B, Zheng X, Lu Y, Rogers T, Osobamiro O et al. Exosomes from marrow stromal cells expressing miR-146b inhibit glioma growth. Cancer Lett 2013; 335: 201-204.

4 Kosaka N, Takeshita F, Yoshioka Y, Hagiwara K, Katsuda T, Ono M et al. Exosomal tumor-suppressive microRNAs as novel cancer therapy: 'exocure' is another choice for cancer treatment. Adv Drug Deliv Rev 2013; 65: 376-382.

5 Fabbri M, Paone A, Calore F, Galli R, Gaudio E, Santhanam R et al. MicroRNAs bind to Toll-like receptors to induce prometastatic inflammatory response. Proc Natl Acad Sci USA 2012; 109: E2110-E2116.

$6 \mathrm{He}$ S, Chu J, Wu LC, Mao H, Peng Y, Alvarez-Breckenridge CA et al. MicroRNAs activate natural killer cells through Toll-like receptor signaling. Blood 2013; 121: 4663-4671.

7 Rana S, Malinowska K, Zoller M. Exosomal tumor microRNA modulates premetastatic organ cells. Neoplasia 2013; 15: 281-295.

8 Tadokoro H, Umezu T, Ohyashiki K, Hirano T, Ohyashiki JH. Exosomes derived from hypoxic leukemia cells enhance tube formation in endothelial cells. J Biol Chem 2013; 288: 34343-34351.

9 Hou W, Zhang Q, Yan Z, Chen R, Zeh lii HJ, Kang R et al. Strange attractors: DAMPs and autophagy link tumor cell death and immunity. Cell Death Dis 2013; 4: e966. 